\title{
EFECTO DEL RALEO EN EL CRECIMIENTO Y ALGUNAS PROPIEDADES DE LA MADERA DE Eucalyptus nitens EN UNA PLANTACIÓN DE 15 AÑOS
}

\section{EFFECT OF THINNING ON GROWTH AND SOME PROPERTIES OF WOOD OF Eucalyptus nitens IN A PLANTATION OF 15 YEARS OLD}

\author{
Sandro Díaz Bravo ${ }^{1 \star}$, Miguel Espinosa ${ }^{2}$, Luis Valenzuela ${ }^{2}$, Jorge Cancino ${ }^{2}$, Jean P. Lasserre ${ }^{1}$
}

\section{RESUMEN}

El raleo tiene implicancias económicas y técnicas tanto para producción forestal como para la actividad industrial. En el presente estudio se realizó la evaluación de un ensayo de intensidad de raleo en E. nitens, de 15 años de edad, intervenido a los 7 y 9 años, con densidades residuales de 300, $400,500,700$ y un tratamiento testigo con $1070 \mathrm{arb} \mathrm{ha}^{-1}$. El objetivo es conocer el efecto del raleo en el diámetro y altura de los árboles, coeficiente de esbeltez, en los índices de competencia área potencialmente aprovechable (APA), área de proyección de copas (APC) y en las propiedades de la madera, densidad básica, módulo de elasticidad dinámico (MOEd) y las tensiones de crecimiento evaluadas con la deformación residual longitudinal de la fibra (DRL).

Los resultados mostraron que el raleo afectó el diámetro, coeficiente de esbeltez, APA y APC, no así la altura. El raleo tampoco tuvo efecto en la densidad básica de la madera y en el DRL. Por otra parte el MOEd fue afectado por el raleo, pero sin mostrar claras tendencias respecto de su intensidad. El efecto de la orientación del fuste en el MOEd y el DRL, mostró valores significativamente mayores en la orientación Sur.

Palabras clave: E. nitens, raleo, densidad básica, módulo de elasticidad, tensiones de crecimiento.

\begin{abstract}
Thinning has significant technical and economic implications for both forest managers and industrial end users. In the present study a 15 year old Eucalyptus nitens thinning experiment was evaluated. Thinnings operations were carried out at 7 and 9 years, with final stockings of 300,400 , 500, 700 and a control treatment of 1070 trees $^{-1} \mathrm{a}^{-1}$ The aim was to determine the effect of thinning on (i) stem diameter and tree height, stem slenderness ratio, (ii) competition indices: potentially usefull area (PUA), crown projection area (CPA) and (iii) wood properties: basic density, dynamic modulus of elasticity (Moed) and growth stresses evaluated using the residual deformation of the fiber length (DRL).

The results showed that thinning affected tree diameter, slenderness index, PUA and CPA, but not total height. Thinning had no effect on basic density and DRL. On the other hand Moed was affected by thinning, but did not show a clear trend with thinning intensity. The effect of the stem orientation on DRL and Moed showed significantly higher values on the South side.
\end{abstract}

Keywords: E. nitens, thinning, basic density, modulus of elasticity, growth stress.

\footnotetext{
${ }^{1}$ Forestal Mininco S.A. Casilla 43-C, Concepción, Chile

${ }^{2}$ Facultad de Ciencias Forestales. Universidad de Concepción, Concepción. Chile.

Autor para correspondencia: ^sandro.diaz@forestal.cmpc.cl

Recibido: 23.06.2011 Aceptado: 28.04.2012
} 


\section{INTRODUCCIÓN}

En Chile existen 168 mil hectáreas plantadas de Eucalyptus nitens (Deane \& Maiden), siendo una de las especies que exhiben las más altas tasas de crecimiento volumétrico de todas las plantaciones comerciales del país (INFOR 2010). Respecto de su uso, aunque E. nitens ha tenido un alto grado de desarrollo y aceptación como materia prima para la fabricación de celulosa, estudios sobre la calidad de su madera para la generación de productos sólidos son aún escasos

En términos generales, E. nitens es considerada una especie con buena aptitud para uso estructural (Nutto y Touza 2004, Nolan et al. 2005, Baker y Volker 2007) cuando es medida a través del módulo de elasticidad (Roth et al. 2007). Por otro lado, con respecto a la densidad básica de la madera, que se relaciona con rendimientos y propiedades de la pulpa y el papel (Megraw 1986, Kennedy 1995), E. nitens es considerada una especie de densidad media. Sin embargo, para usos sólidos, entre las principales limitaciones de la madera de $E$. nitens, destacan su alta variabilidad física y mecánica, además de defectos generados por altas tensiones de crecimiento que se manifiestan en rajaduras, colapso y grietas que disminuyen el aprovechamiento de la madera (Valencia 2008).

El manejo silvícola con podas y/o raleos, ha sido una de las herramientas que los silvicultores han utilizado en E. nitens para lograr una mayor diversificación de productos y por lo tanto aumentar el valor del patrimonio forestal. Aunque la superficie manejada de $E$. nitens ha aumentado lenta pero sostenidamente en el país (INFOR 2007), son escasos los estudios que evalúan el efecto del manejo en el crecimiento de los árboles (Stöckle 1996, Celhay et al. 1999, Muñoz et al. 2005) y menos aún, los que relacionan el efecto del raleo en las propiedades de la madera.

Dado que el conocimiento de los factores que influyen en la calidad de la madera, es vital para mejorar el valor de las plantaciones (Sarén et al. 2004), los objetivos de este estudio fueron: a) evaluar el efecto del raleo en el desarrollo en diámetro, altura, coeficiente de esbeltez y los índices de competencia área potencialmente aprovechable y área de proyección de copas; b) evaluar el efecto del raleo en la densidad básica de la madera, en el módulo de elasticidad dinámico y en las tensiones de crecimiento; c) evaluar el efecto de la orientación del fuste en las propiedades de la madera y d) relacionar variables de estado e índices de competencia de los tratamientos de raleo con las propiedades de la madera.

\section{MATERIAL Y MÉTODOS}

\section{Características del sitio}

El área de estudio se ubica en el Fundo Huallenco de propiedad de Forestal Mininco S.A., localizado en la precordillera andina de Mulchén, Región del Biobío, Chile ( $37^{\circ} 51^{\prime} \mathrm{S}$ y $72^{\circ} 07^{\prime} \mathrm{O}$ ), a una altitud de $340 \mathrm{~m}$ s.n.m. Tiene una topografía plana y pendientes inferiores a 7\%. La precipitación promedio anual supera los $1000 \mathrm{~mm}$, distribuidos en un $80 \%$ entre los meses de abril a octubre. Las temperaturas promedio oscilan entre los $5{ }^{\circ} \mathrm{C}$ la mínima y los $21^{\circ} \mathrm{C}$ la máxima (registro histórico estación meteorológica de Forestal Mininco S.A.).

El suelo es un trumao derivado de cenizas volcánicas, profundo, con drenaje interno moderado a rápido, generalmente rico en materia orgánica y de buena productividad (Schlatter et al. 1994). 


\section{Descripción del ensayo}

El efecto del raleo en el crecimiento de los árboles y algunas propiedades de la madera, se evaluó en una plantación de E. nitens de 15 años de edad, en 18 parcelas experimentales de $1000 \mathrm{~m}^{2}\left(31.6^{*}\right.$ $31.6 \mathrm{~m}$ ), cada una de ellas rodeada de un área buffer de $10 \mathrm{~m}$, con el mismo tratamiento para evitar el efecto de borde. Las parcelas se establecieron a los siete años de edad, según un diseño de bloques completos al azar con tres repeticiones. Los raleos fueron ejecutados a los siete y a los nueve años, con las siguientes densidades residuales (Tabla 1).

Tabla 1. Descripción del ensayo y sus tratamientos.

\begin{tabular}{ll}
\hline Código & Tratamiento \\
\hline T1 & 300 árboles ha ${ }^{-1}$, con raleo a los 7 años \\
T2 & 500 árboles $\mathrm{ha}^{-1}$, con raleo a los 7 años \\
T3 & 700 árboles $\mathrm{ha}^{-1}$, con raleo a los 7 años \\
T4 & 300 árboles $\mathrm{ha}^{-1}$, con raleo a los 7 y 9 años \\
T5 & 400 árboles $\mathrm{ha}^{-1}$, con raleo a los 7 y 9 años \\
T6 & 1070 árboles $\mathrm{ha}^{-1}$, sin raleo (Testigo) \\
\hline
\end{tabular}

\section{Medición de variables y cálculos}

Para determinar el efecto del raleo en el crecimiento de los árboles y en las propiedades de la madera, densidad básica, módulo de elasticidad dinámico y en las tensiones de crecimiento, se usó una muestra de 10 árboles del área buffer por parcela, distribuidos por clase diamétrica (30 individuos por tratamiento, 180 árboles en total). A cada árbol se le midió el diámetro a la altura del pecho (Dap) y la altura total y con estas dos variables se calculó el coeficiente de esbeltez (altura/Dap). Adicionalmente se midieron los índices de competencia, Área Potencialmente Aprovechable (APA) y el Área de Proyección de Copas (APC) con la finalidad de establecer relaciones con variables de estado (Dap, altura y esbeltez) y con las propiedades de la madera evaluadas.

\section{Medición de APA y APC}

El APA se determinó midiendo el rumbo y la distancia entre un árbol y sus vecinos más cercanos, formando un polígono alrededor del árbol, cuyos lados son rectas trazadas entre los árboles competidores inmediatos y el árbol muestreado. Las intersecciones entre estas rectas definen los vértices del polígono, de manera que la suma de sus áreas es igual al total de la superficie (Prodan et al. 1997), como se muestra en la figura 1 . 


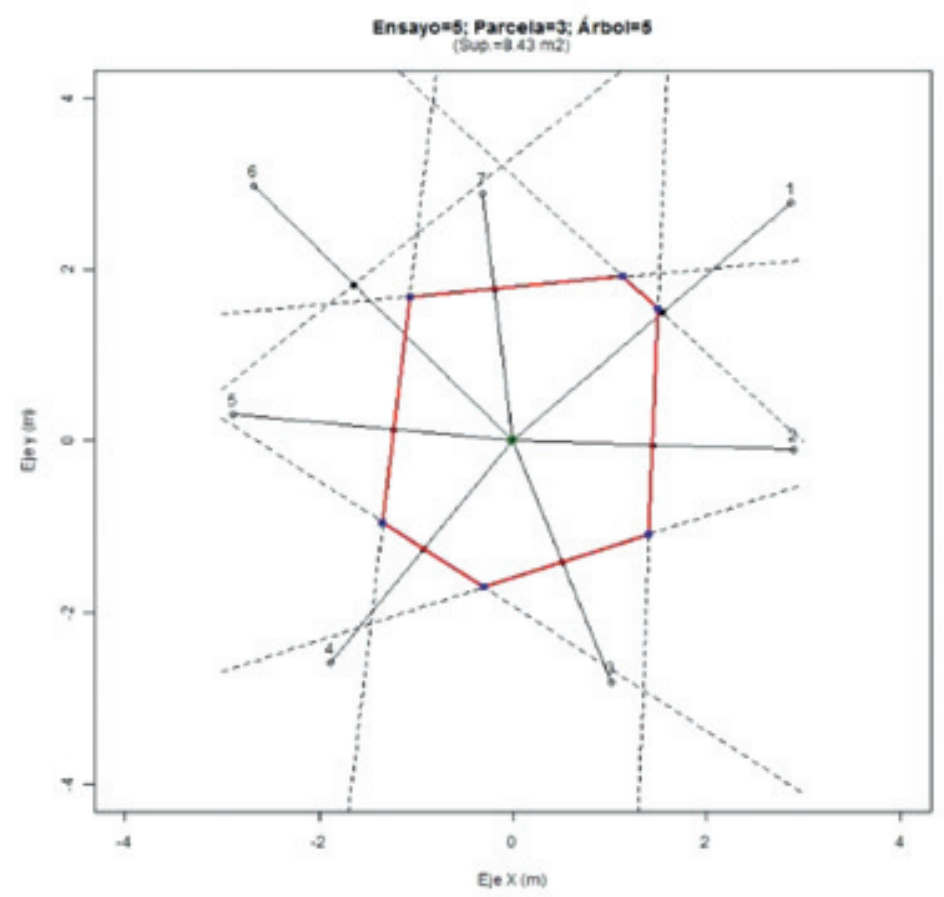

Figura 1. Representación gráfica de la medición del área potencialmente aprovechable.

Para determinar el APC se midió la distancia desde el centro del fuste hasta donde termina la proyección de la copa de cada árbol, en los cuatro sentidos cardinales. Estas medidas se hicieron con el apoyo de un espejo fijado en una superficie de madera lisa que tiene adheridos en sus bordes dos niveles. Con las distancias se calculó el APC en $\mathrm{m}^{2}$, mediante la expresión:

$$
\mathrm{APC}=\pi^{*} \mathrm{r}^{2}
$$

Donde " $r$ " es el promedio de las distancias N-S-E-W.

\section{Medición de la densidad verde y densidad básica de la madera}

Para determinar la densidad verde y densidad básica de la madera, en cada árbol se sacaron tarugos de $12 \mathrm{~mm}$, en dirección Norte-Sur a la altura del Dap (corteza-médula-corteza) y se enviaron al laboratorio en un tubo sellado, para luego pesarlos y posteriormente medir su volumen por desplazamiento de agua, siguiendo las recomendaciones del método B de la norma americana ASTM D-2395.

\section{Medición del módulo de elasticidad dinámico (MOEd)}

En árboles en pie se midió el tiempo de vuelo (Tv) de una onda acústica entre dos puntos de distancia conocida ( $l$ y y mediante la relación $l / T v$, se obtuvo la velocidad de la onda acústica $(\mathrm{V})$. Con este valor, más la densidad verde $(r)$, se obtuvo el MOEd mediante la siguiente expresión:

$$
\operatorname{MOEd}=\rho \mathrm{V}^{2}
$$

El Tv se midió con el instrumento portátil FAKOPP, realizando dos lecturas en cada árbol, una sobre la cara Norte del fuste y otra sobre la cara Sur. Los sensores acústicos se ubicaron a una altura 
de $1 \mathrm{~m}$ desde el suelo y a $1 \mathrm{~m}$ de distancia entre sí. Este mismo método llamado "tiempo de vuelo", ha sido usado por otros autores (Chauhan y Walker 2006, Lasserre et al. 2008), entre otros.

\section{Medición de tensiones de crecimiento}

Las tensiones de crecimiento se midieron a todos los árboles con un instrumento denominado Extensómetro, siguiendo el protocolo desarrollado por el centro de investigación CIRAD-Forêt, de Francia y utilizado por varios investigadores (Valdes 2004, Yang et al. 2005, Valencia 2008). Se realizaron dos mediciones opuestas por árbol, aplicando el procedimiento a la altura del Dap y definiendo la orientación de una primera ventana en sentido Norte y la otra hacia el Sur. Las mediciones fueron hechas en verano con un protocolo muy riguroso que considera la medición sin viento, permitiendo la estabilidad vertical del árbol sin afectar la medición del instrumento. Aquí se midió el desplazamiento de la fibra en dirección longitudinal, como una medida directa de la tensión liberada y denominada deformación residual longitudinal (DRL), en milímetros.

\section{Análisis de datos}

Se realizó un análisis estadístico utilizando el programa SAS (SAS Institute, 2000), relacionando todas las variables de estado e índices de competencia y las propiedades de la madera, con cinco de las seis densidades residuales consideradas (300, 400, 500, 700 y testigo). Los tratamientos con 300 arb ha ${ }^{-1}$ de densidad residual, en uno ó dos raleos, se agruparon debido a que no presentaron diferencias significativas $(\mathrm{p}<=0.05)$.

Se aplicó análisis de varianza para detectar evidencias de diferencias estadísticas entre los tratamientos, utilizando el test de Tukey para separar las medias cuando los efectos mostraron ser significativos ( $\mathrm{p}<=0.05$ ). Se realizó el análisis de varianza de cada variable por separado y luego se determinó el grado de relación entre las variables, con la matriz de correlación de Pearson.

\section{RESULTADOS Y DISCUSIÓN}

\section{Efecto del raleo en las variables de estado e índices de competencia}

El promedio de las mediciones de Dap, altura, coeficiente de esbeltez, APA y APC, con sus correspondientes desviaciones estándar (D.E.), se presentan en la tabla 2.

Tabla 2. Variables de estado e índices de competencia, en E. nitens de 15 años.

\begin{tabular}{|c|c|c|c|c|c|c|c|c|c|c|}
\hline $\begin{array}{c}\text { Densidad } \\
\text { residual } \\
\left(\text { arb ha }{ }^{-1}\right)\end{array}$ & $\begin{array}{l}\text { Dap } \\
(\mathrm{cm})\end{array}$ & $\begin{array}{l}\text { D.E } \\
\text { (cm) }\end{array}$ & $\begin{array}{l}\text { Altura } \\
\text { (m) }\end{array}$ & $\begin{array}{l}\text { D.E. } \\
\text { (m) }\end{array}$ & $\begin{array}{l}\text { C. Esbeltez } \\
\left(\mathrm{m} \mathrm{cm}^{-1}\right)\end{array}$ & $\begin{array}{c}\text { D.E. } \\
\left(\mathrm{m} \mathrm{cm}^{-1}\right)\end{array}$ & $\begin{array}{l}\text { APA } \\
\left(\mathrm{m}^{2}\right)\end{array}$ & $\begin{array}{l}\text { D.E. } \\
\left(\mathrm{m}^{2}\right)\end{array}$ & $\begin{array}{l}\text { APC } \\
\left(\mathrm{m}^{2}\right)\end{array}$ & $\begin{array}{l}\text { D.E. } \\
\left(\mathrm{m}^{2}\right)\end{array}$ \\
\hline 300 & $\underline{42,0}^{\mathrm{a}}$ & 6,6 & $\underline{41,2}^{\mathrm{a}}$ & 3,7 & $1,00^{b}$ & 0,14 & $\underline{36,4^{2}}$ & 12,5 & $22,3^{2}$ & 8,6 \\
\hline 400 & $38,6^{\text {ab }}$ & 6,6 & $40,6^{a}$ & 4,0 & $1,07^{b}$ & 0,14 & $29,6^{b}$ & 13,5 & $22,5^{a}$ & 9,5 \\
\hline 500 & $35,7^{\mathrm{bc}}$ & 7,7 & $40,8^{a}$ & 4,9 & $1,17^{\mathrm{a}}$ & 0,18 & $20,2^{\circ}$ & 7,5 & $\overline{14,9}^{b}$ & 7,0 \\
\hline 700 & $34,9^{\mathrm{bc}}$ & 7,1 & $39,6^{a}$ & 4,5 & $1,17^{a}$ & 0,22 & $15,9^{\text {cd }}$ & 5,9 & $13,7^{b}$ & 6,1 \\
\hline Testigo & $32,7^{\mathrm{c}}$ & 7,1 & $39,3^{\text {a }}$ & 4,9 & $1,24^{\mathrm{a}}$ & 0,22 & $12,0^{\mathrm{d}}$ & 3,4 & $11,1^{\mathrm{b}}$ & 5,3 \\
\hline
\end{tabular}

Nota $1:$ Dap = Diámetro a la altura del pecho a 1,3 m, D.E.= Desviación estándar, C. Esbeltez = Coeficiente de esbeltez, APA = Área potencialmente aprovechable, APC = Área de proyección de copa.

Nota 2 : Letras distintas indican diferencias significativas $(\mathrm{p}<=0.05)$. En cada columna se destacan en negrilla aquellos tratamientos que no presentan diferencias significativas con el mayor valor observado (subrayado). 
El Dap mostró una tendencia a disminuir a medida que la densidad residual es mayor. Los dos tratamientos más intensamente raleados (300 y $400 \mathrm{arb} \mathrm{ha}^{-1}$ residuales) fueron estadísticamente diferentes respecto al Dap del testigo, alcanzando valores promedio mayores de 9,3 y $5,9 \mathrm{~cm}$, respectivamente (Tabla 2 ).

El resultado mencionado anteriormente, fue consistente con otros estudios para E. nitens (Neilsen y Pinkard 1999, Medhurst y Beadle 2000, Medhurst et al. 2001, Muñoz et al. 2005, Nolan et al. 2005) en los que se demostró que el mayor incremento en Dap se encontró en los tratamientos más intensamente raleados. Así también, otros estudios demuestran que los raleos alteran las condiciones medioambientales necesarias para el crecimiento, incrementando la disponibilidad de agua, luz y nutrientes para los árboles remanentes (Medhurst et al. 2002).

El crecimiento en altura de los árboles no se aprecio afectado por la intensidad del raleo (Tabla 2), esto es consistente con lo registrado en otros estudios (Medhurst et al. 2001, Muñoz et al. 2005), sin embargo, tiene una tendencia decreciente con la densidad residual, registrándose diferencias de casi dos metros de altura, entre el tratamiento de menor densidad residual y el testigo (Tabla 2).

El coeficiente de esbeltez (razón altura/diámetro), es estadísticamente menor en los tratamientos de 300 y $400 \mathrm{arb} \mathrm{ha}^{-1}$, respecto de los tratamientos con mayor densidad residual y el testigo (Tabla 2). Dado que el coeficiente de esbeltez es función de la densidad y que el raleo afecta en mayor medida el crecimiento en diámetro que en altura, éste tiende a aumentar a medida que aumenta la densidad residual. Mantener la razón altura/diámetro bajo 1, se estima reduce la susceptibilidad de los árboles a daños por viento y nieve (Cremer et al. 1982, Wilson y Oliver 2000). Por ello, este coeficiente se considera un buen indicador para prescribir la oportunidad de los raleos: a mayor esbeltez los árboles son más susceptibles a sufrir daños por viento, por lo tanto, es una variable que condiciona la intensidad del raleo.

Como era de esperar, el índice de competencia para árboles individuales, área potencialmente aprovechable (APA), tiende a aumentar a medida que la densidad disminuye. Los árboles del tratamiento con $300 \mathrm{arb} \mathrm{ha}^{-1}$ tienen un APA promedio de $36.4 \mathrm{~m}^{2}$, valor significativamente mayor que los restantes tratamientos de raleo y tres veces superior a los $12.0 \mathrm{~m}^{2}$ del testigo (Tabla 2).

El raleo también tuvo un efecto significativo en el área de proyección de la copa (APC). En promedio, cada árbol de los tratamientos con 300 y $400 \mathrm{arb} \mathrm{ha}^{-1}$, cubren más de $22.3 \mathrm{~m}^{2}$ de terreno con su copa, mientras el testigo sólo $11.2 \mathrm{~m}^{2}$ (Tabla 2), 100\% menos de cobertura por árbol. Las mayores dimensiones de los árboles de las parcelas más intensamente raleadas explican estos resultados.

La eficiencia de uso del sitio como índice de competencia, se puede analizar con la relación APC/ APA, que proporciona un indicador relativo de cómo las copas son capaces de usar la mayor cantidad de espacio disponible, dependiendo de la intensidad del raleo. En este estudio los valores varían desde $61 \%$ en el caso de $300 \mathrm{arb} \mathrm{ha}^{-1}$, hasta 93\% en las parcelas testigo, lo que indicaría un dosel cerrado o una ocupación casi total del área aprovechable de cada árbol y por consiguiente mayor competencia y menor crecimiento individual.

\section{Efecto del raleo en las propiedades de la madera}

Las propiedades de la madera densidad básica, MOEd y las tensiones de crecimiento, evaluadas a través de la deformación residual longitudinal de la fibra (DRL) y sus correspondientes desviaciones estándar (D.E.), se presentan en la tabla 3. Para la densidad básica de la madera se presenta el valor promedio de los tarugos, por tratamiento. Los valores de MOEd y DRL, corresponden al promedio de las mediciones Norte-Sur, por árbol y tratamiento, más adelante se analizará el efecto de la orientación del fuste. 
Tabla 3. Propiedades de la madera de E. nitens de 15 años de edad.

\begin{tabular}{crrrrrr}
\hline $\begin{array}{c}\text { Densidad } \\
\text { residual } \\
\left(\text { arb ha }^{-1}\right)\end{array}$ & D. Básica $\left(\mathrm{kg} \mathrm{m}^{-3}\right)$ & \multicolumn{2}{c}{ MOEd $(\mathrm{GPa})$} & \multicolumn{2}{c}{ DRL $(\mathrm{mm})$} \\
\cline { 2 - 7 } & Promedio & D.E. & Promedio & D.E. & Promedio & D.E. \\
\hline 300 & $\mathbf{4 8 9 , 7}^{\text {a }}$ & 36,4 & $15,4^{\text {b }}$ & 1,9 & $\underline{\mathbf{0 , 1 5 6}}^{\text {a }}$ & 0,077 \\
400 & $\mathbf{4 7 3 , 4}^{\text {a }}$ & 28,1 & $15,0^{\text {b }}$ & 2,2 & $\mathbf{0 , 1 5 2}^{\text {a }}$ & 0,078 \\
500 & $\mathbf{4 7 6 , 6}^{\text {a }}$ & 35,8 & $15,5^{\text {b }}$ & 2,0 & $\mathbf{0 , 1 3 8}^{\text {a }}$ & 0,070 \\
700 & $\mathbf{4 9 2 , 2}^{\text {a }}$ & 36,7 & $\underline{\mathbf{1 6 , 3}}^{\text {a }}$ & 2,2 & $\mathbf{0 , 1 4 6}^{\text {a }}$ & 0,069 \\
Testigo & $\mathbf{4 8 4 , 2}^{\text {a }}$ & 29,2 & $15,5^{\text {b }}$ & 2,1 & $\mathbf{0 , 1 4 1}$ & 0,079 \\
\hline
\end{tabular}

Nota $1:$ D. Básica = Densidad básica de la madera, MOEd = Módulo de elasticidad dinámico, DRL $=$ Deformación residual longitudinal, D.E.= Desviación estándar.

Nota 2 : Letras distintas indican diferencias significativas $(\mathrm{p}<=0.05)$. En cada columna se destacan en negrilla aquellos tratamientos que no presentan diferencias significativas con el mayor valor observado (subrayado).

\section{Densidad básica de la madera}

La densidad básica no presentó diferencias significativas entre tratamientos, con valores promedio que varían entre 473,4 y 492,2 $\mathrm{kg} \mathrm{m}^{-3}$ (Tabla 3), lo que indicaría que el raleo no afectaría esta variable, importante para su uso industrial, por su relación directa con el rendimiento pulpable de la madera.

El efecto del espaciamiento y los raleos sobre latifoliadas ha sido poco reportado; Higgs (1969) en E. grandis encontró mayores largos de fibra pero sin cambios en densidad básica. En la misma especie Schönau (1974), indica que la densidad básica no fue afectada por el espaciamiento. En E. saligna los cambios en densidad residual no afectaron la densidad básica (Guimaraes 1965, Ferreira 1968, Zobel y Sprague 1998). En E. nitens, Muñoz et al. (2010), indica que la densidad básica promedio del rodal no es afectada por la densidad residual del rodal.

Según Zobel y Van Buijtenen (1989) el efecto de los raleos en la densidad básica de la madera es importante en los primeros 4 años después del raleo. En este estudio se evaluó el efecto del raleo 8 y 6 años después de efectuada esta intervención, sin registrarse diferencias significativas.

En este ensayo para E. nitens de 15 años de edad, el promedio de densidad básica de todos los tratamientos es de $483 \mathrm{~kg} \mathrm{~m}^{-3}$ con una desviación estándar de $33 \mathrm{~kg} \mathrm{~m}^{-3}$, valor similar a otros estudios relacionados, en la misma especie (Farrel et al. 2010).

\section{Módulo de elasticidad dinámico (MOEd)}

La intensidad de raleo no tiene un efecto claro de aumentar o disminuir el MOEd . Sólo el tratamiento de 700 arb ha-1 ${ }^{-1}$ resentó diferencias significativas en el MOEd con un valor promedio de 16,3 $\mathrm{GPa}$ (Tabla 3). Este valor, así como el de los restantes tratamientos que varían entre 15,0 y 15,5 GPa, son altos comparados con los reportados en otras publicaciones (Yang y Evans 2003, Valencia 2008, Farrel et al. 2008).

Warren et al. (2009) determinaron que el MOEd, en tres especies de eucalipto evaluado a los seis

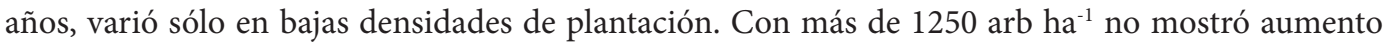
significativo en la rigidez de los árboles . Por el contrario, Waghorn et al. (2007) determinaron que el MOEd aumentó con la densidad de 5,1 a 7,0 GPa entre 275 y 2551 arb ha $^{-1}$ en pino radiata de 17 años. 
La misma tendencia encontró Lasserre et al. (2008) en rodales de esta especie de 11 años de edad, evaluando dos sitios, tres clones y dos densidades (833 y 2500 arb ha $^{-1}$ ) y también Valencia (2008) en E. nitens de 22 años.

El MOEd a lo largo de la fibra, que es una función de la velocidad de la onda acústica y la densidad de la madera, se ha demostrado que se correlaciona bien con el MOE estático (Carter et al. 2005). Sin embargo, esta onda acústica viaja en forma tangencial entre ambos puntos (emisor y receptor) por lo que el sistema adolece de una imprecisión en la determinación de la propiedad de la madera en la parte central del trozo, aún así, es un buen indicador de la aptitud estructural de la madera, superando en todos los tratamientos el límite inferior de $10 \mathrm{GPa}$ (Farrel et al. 2008).

\section{Deformación residual longitudinal (DRL)}

Las tensiones de crecimiento no pueden ser medidas en forma directa, es necesario medir el DRL y el MOEd, sin embargo, según De Fégely (2004) y Raymond et al. (2004), el DRL por si solo es un buen indicador de las tensiones de crecimiento de los árboles.

El DRL no presenta diferencias significativas entre las densidades residuales evaluadas (Tabla 3), lo que indicaría que las intensidades de raleo consideradas no afectarían las tensiones de crecimiento de los árboles, característica de la madera de E. nitens que se manifiesta en rajaduras, colapso y grietas que disminuyen el aprovechamiento de la madera.

Este resultado coincide con lo registrado por Valencia (2008) en E. nitens de 22 años, creciendo en Tasmania Australia, quien no encontró influencia del raleo en las tensiones de crecimiento. Sin embargo, Ferrand (1983) determinó bajos niveles de DRL en tratamientos intensamente raleados, no así en raleos de baja intensidad.

Los valores de DRL de este estudio son menores a los encontrados por Valdés (2004) en E. nitens de 13 años de edad, que compara 10 familias en dos huertos semilleros de distinto sitio, con un promedio de 0,244 mm, teniendo ambos huertos aproximadamente $400 \mathrm{arb} \mathrm{ha}^{-1}$. En su estudio Valdés encontró diferencias entre familias, no así entre los sitios. De igual forma, Mutizabal (2007) no encontró diferencias entre el nivel de tensiones de tres procedencias de E. nitens (Toorongo, Tallaganda y Errinundra) de 17 años y 200 arb ha $^{-1}$, con raleo a los cinco años de edad.

Esta variabilidad de resultados puede ser atribuida a factores genéticos, tratamientos silviculturales, condiciones del medio ambiente como sitio, elevación y exposición al viento, entre otras (Chauhan 2004). Nolan et al. (2005) sugieren que la reducción de las tensiones de crecimiento debe ser un objetivo importante en las plantaciones gestionadas para productos de madera sólida.

\section{Efecto de la orientación del fuste, respecto de las propiedades de la madera}

El efecto de la orientación del fuste en el MOEd promedio y su correspondiente error estándar de medición, para cada tratamiento de raleo, en los sentidos Norte y Sur, se presenta en la figura 2. 


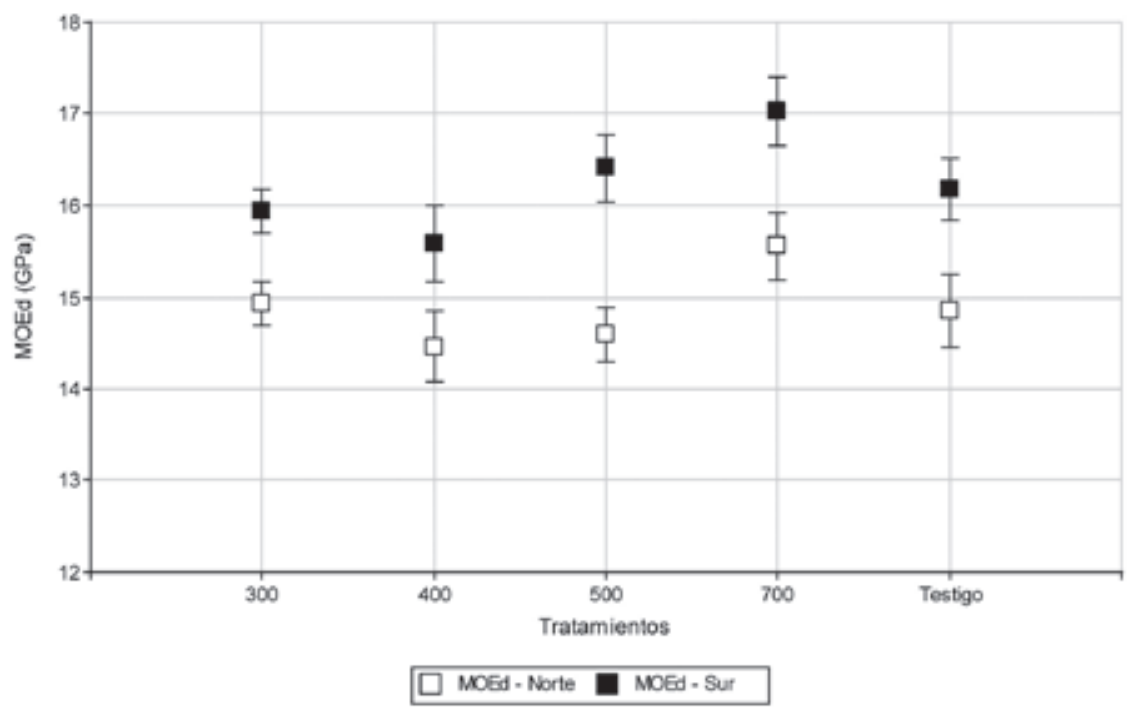

Figura 2. Módulo de elasticidad dinámico según tratamiento y orientación del fuste.

De esta figura se puede observar que la intensidad de raleo no tiene un efecto claro de aumentar o disminuir el MOEd, sin embargo sí se encontró diferencias significativas entre la orientación Norte y Sur ( $\mathrm{p}<=0.05$ ). Esta siempre es mayor en el sentido Sur (Figura 2), con un promedio de 16,2 GPa, que en el Norte con 14,9 GPa. La diferencia es un 8,7\% más de MOEd en el Sur.

Bascuñan (2004), en pino radiata, encontró que las diferencias en la rigidez de la madera entre el lado de sotavento (sentido contrario a los vientos dominantes) y barlovento (sentido de los vientos dominantes) de un árbol, pueden ir desde cero hasta un máximo de 55\%. Estas diferencias aumentan con la edad de los árboles, la inclinación de los fustes y si el árbol está situado en el borde del rodal. Encontró que en promedio el lado de sotavento de los árboles presenta una mayor rigidez que el lado de barlovento. Los valores de la rigidez de sotavento fueron entre 10 a 18\% más altos que los del fuste de barlovento.

Estos resultados concuerdan con las observaciones formuladas por Grabianowski (2003), quien hizo una comparación entre las velocidades acústicas de los diferentes lados de 11 árboles de pino radiata de 27 años de edad, donde encontró diferencias entre el lado sureste del fuste, con mayores velocidades que los lados noreste y noroeste, respectivamente. En su estudio, el lado sureste (sotavento), es el lado donde era esperable madera de compresión debido al viento predominante del noroeste (barlovento).

Por el contrario, los resultados de este estudio, muestran una tendencia clara y con diferencias significativas ( $\mathrm{p}<=0.05)$, arrojando siempre mayores valores en la cara Sur (barlovento), debido probablemente a la formación de madera de reacción. Según estos resultados, el comportamiento del MOEd o los niveles de velocidad acústica en E. nitens se producirían en orientaciones contrarias a los producidos en pino radiata.

Valencia (2008) encontró diferencias significativas en los niveles de velocidad acústica en E. nitens, con la misma tendencia que este estudio: mayor en la cara de la dirección del viento dominante. 
Se ha planteado la hipótesis de que bajas densidades residuales permiten una mayor influencia del viento dentro del bosque y que a su vez esto podría dar lugar a que la rigidez de la madera disminuya. Sin embargo, poco se sabe sobre el efecto del viento en la regulación de la rigidez de los árboles, en particular en especies de Eucalyptus (Warren et al. 2009).

El efecto de la orientación del fuste en las tensiones de crecimiento evaluadas a través de la DRL promedio y su correspondiente error estándar de medición, para cada tratamiento de raleo, en los sentidos Norte y Sur, se presentan en la figura 3.

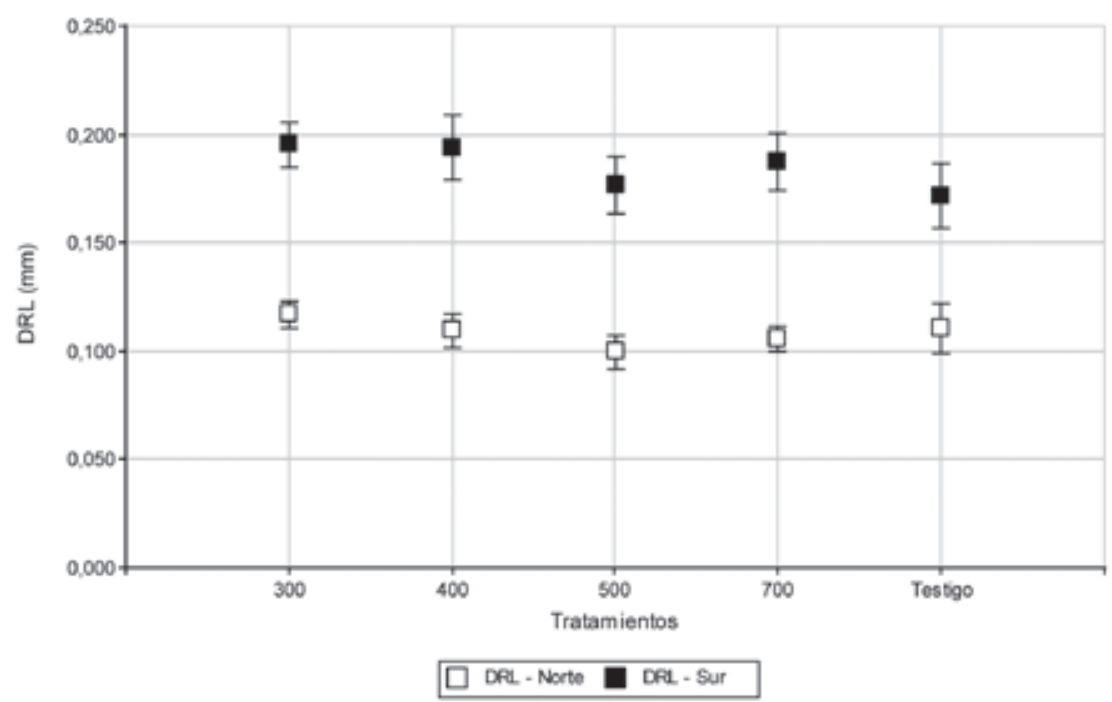

Figura 3. Deformación residual longitudinal según tratamiento y orientación del fuste.

El análisis estadístico no mostró diferencias significativas entre tratamientos de raleo, pero sí respecto a la orientación Norte y Sur $(\mathrm{p}<=0.05)$, siendo mayores los niveles de tensión liberada en el sentido Sur (Figura 3), con un promedio de $0.185 \mathrm{~mm}$, que en el Norte, con $0.108 \mathrm{~mm}$. La diferencia corresponde a un $70.6 \%$ más de DRL en el Sur.

Valencia et al. (2011) encontraron diferencias significativas del DRL en E. nitens de 22 años de edad, en Tasmania, Australia, con la misma tendencia que este estudio, siendo mayor en la cara de la dirección del viento dominante.

Kenneth (2001) establece que uno de los orígenes de las tensiones de crecimiento estaría en la formación de madera de reacción, en aquellos casos donde el hábito natural de crecimiento vertical de los árboles se ve alterado, ya sea por viento o inclinación del fuste. Como respuesta a la distribución asimétrica de fuerzas dentro del fuste, el cambium produce madera de reacción en diferentes direcciones, según se trate de coníferas o latifoliadas.

En latifoliadas la madera de reacción, denominada madera de tensión, ocurre en el lado expuesto a las fuerzas que inclinan el árbol, produciendo fustes excéntricos debido a la tensión contráctil del fuste, para mantener la posición vertical del ápice (Telewski 1995, Kenneth 2001). 
Otro factor que ha sido reportado como responsable del incremento de las tensiones de crecimiento en alguna orientación del fuste en diferentes especies de Eucalyptus, es la asimetría de las copas, que puede incrementar el estrés de crecimiento en una cara del fuste, por lo tanto esto podría explicar los niveles más altos de DRL en la orientación del viento predominante (Raymond et al. 2004, Yang 2005, Cardoso et al. 2005, Trugilho et al. 2006).

La diferencia de DRL encontrada en este estudio entre las orientaciones Norte y Sur, recomienda generar nuevas investigaciones para evaluar el efecto de la madera de reacción (tensión) y las tensiones de crecimiento, relacionando principalmente variables ambientales (viento, entre otras) y de manejo silvicultural (espaciamiento inicial, asimetría de las copas, intensidad y oportunidad de raleos) con el proceso de aserrío y secado de la madera de E. nitens.

\section{Relaciones entre variables de estado, índices de competencia y propiedades de la madera}

En general la altura, el coeficiente de esbeltez, el APA y el APC tienen alto grado de asociación lineal con el Dap, con coeficientes de correlación sobre 0.6 (Tabla 4). Esta relación es lógica, al igual que el alto grado de correlación de los índices APA y APC, entre sí y con el coeficiente de esbeltez, ya que el diámetro es determinado por la competencia de los árboles, no así la altura, con bajas correlaciones.

En términos generales en $E$. nitens hay pocos reportes que relacionen la esbeltez con la calidad de la madera; en pino radiata, Watt et al. (2006) encontró relación entre esbeltez y algunas propiedades de la madera, principalmente con MOE.

Una relación positiva significativa encontraron Lasserre et al. (2005) entre la densidad residual y la velocidad acústica medida en árboles en pie de pino radiata. La velocidad acústica y la rigidez de la madera fueron influenciadas positivamente por la densidad de plantación y negativamente por el diámetro. La misma tendencia ha sido mencionada por Carter et al. (2005) y Chauhan y Walker (2006), con menor velocidad acústica en la medida que aumenta el diámetro. En este estudio el MOEd no presenta relación lineal con el diámetro.

De acuerdo a estos resultados, las propiedades de la madera evaluadas no tienen asociación lineal con respecto a las variables de estado e índices de competencia considerados. Las correlaciones MOEd v/s DRL y MOEd v/s D. Básica fueron significativas al 99.9\% y 99.0\% de nivel de confianza, respectivamente (Tabla 4$)$.

En otros estudios, Trugilho et al. (2004) y Valdés (2004) encontraron una relación positiva entre la DRL y el diámetro del árbol para 15 y 19 años de edad en E. dunnii y 13 años de edad en E. nitens, respectivamente.

Los resultados de este estudio no mostraron correlaciones significativas entre variables de estado y densidad básica, MOEd y DRL, lo que sería una herramienta práctica para que los silvicultores pudieran manejar sus bosques hacia diferentes usos finales. Sin embargo, el que los tratamientos de raleo no hayan afectado significativamente ninguna de las propiedades de la madera evaluadas, indicaría que, bajo las condiciones de este estudio, el raleo no tiene un efecto negativo en la calidad de la madera. 
Tabla 4. Matriz de correlación de Pearson.

\begin{tabular}{|c|c|c|c|c|c|c|c|}
\hline & Dap & Altura & C. Esbeltez & $\mathrm{APA}$ & $\mathrm{APC}$ & DRL & MOEd \\
\hline Altura & $0,60^{\cdots \cdots}$ & & & & & & \\
\hline C. Esbeltez & $-0,85^{\cdots *}$ & $-0,13^{\circ}$ & & & & & \\
\hline APA & $0,71^{\cdots *}$ & $0,39^{* *}$ & $-0,611^{\cdots *}$ & & & & \\
\hline APC & $0,77^{\cdots *}$ & $0,41^{* *}$ & $-0,67^{* * *}$ & $0,74^{\cdots *}$ & & & \\
\hline DRL & $0,07^{\mathrm{ns}}$ & $0,05^{\text {ns }}$ & $-0,07^{\text {ns }}$ & $0,03^{\mathrm{ns}}$ & $0,07^{\mathrm{ns}}$ & & \\
\hline MOEd & $-0,02^{n s}$ & $0,07^{\mathrm{ns}}$ & $0,05^{\text {ns }}$ & $-0,08^{\text {ns }}$ & $0,01^{n s}$ & $0,57^{\cdots}$ & \\
\hline D. Básica & $0,08^{\text {ns }}$ & $0,04^{\mathrm{ns}}$ & $-0,09^{\text {ns }}$ & $0,06^{\text {ns }}$ & $0,07^{\mathrm{ns}}$ & $0,16^{\circ}$ & $0,44^{* *}$ \\
\hline
\end{tabular}

Nota 1: Dap = Diámetro a la altura del pecho a 1,3 m, C. Esbeltez = Coeficiente de esbeltez, APA = Área potencialmente aprovechable, APC = Área de proyección de copa, DRL = Deformación residual longitudinal, MOEd = Módulo de elasticidad dinámico, D. Básica $=$ Densidad básica de la madera. Nota 2: ns: no significativo $\mathrm{P}=0.05$.

* Significativo $\mathrm{P}=0.05$.

** Significativo $\mathrm{P}=0.01$.

$* * *$ Significativo $\mathrm{P}=0.001$.

\section{CONCLUSIONES}

El raleo de E. nitens afectó significativamente algunos parámetros de estado del rodal, como el Dap, APA y APC. El Dap presentó un mayor desarrollo en tratamientos de menores densidades residuales, con más de seis $\mathrm{cm}$ de diferencia en promedio con respecto al testigo sin raleo. La altura no presentó diferencias significativas, sin embargo muestra una tendencia decreciente en la medida que el número de árboles residuales aumenta. La razón altura/diámetro o coeficiente de esbeltez aumentó en la medida que la densidad residual es mayor.

Los índices de competencia presentan una tendencia decreciente, con diferencias significativas a favor de los tratamientos más intensamente raleados. El APA del tratamiento más intensamente raleado es tres veces el área del tratamiento testigo. Por su parte el APC de los tratamientos más intensamente raleados, es dos veces superior al Testigo. La relación APC/APA fue afectada por el raleo, mostrando un mayor grado de competencia y menor crecimiento individual, en la medida que aumenta el número de árboles residuales.

Respecto de las propiedades de la madera, el raleo no tuvo efecto en la densidad básica de la madera y en las tensiones de crecimiento. El módulo de elasticidad dinámico sí presentó diferencias significativas, pero sin un efecto claro de aumentar o disminuir esta propiedad, en relación a las densidades residuales evaluadas.

El Dap exhibió una alta correlación con los índices de competencia, sin embargo no mostró una correlación significativa con las propiedades de la madera.

El efecto de la orientación del fuste, en el módulo de elasticidad dinámico y las tensiones de crecimiento, mostró valores significativamente mayores en la orientación Sur. Se requiere mayor investigación respecto del efecto de la orientación y sus causas, ya que podría ser una herramienta muy útil para el manejo de las plantaciones y para relacionar otras variables como el efecto del viento, madera de reacción, evaluar genotipos con mejores propiedades, entre otros. 


\section{BIBLIOGRAFÍA}

ASTM. American Society for Testing and Materials. 1998. Standard Test Methods for Specific Gravity of Wood-Based Materials (D-2395). Annual Book of ASTM Standard. Section 4, Construction v. 0410 Wood. Easton. 666 p.

Baker, T.; Volker, P. 2007. Silviculture of eucalypt plantations in southern Australia for highvalue solid wood products. CIFOR 13: 43-57.

Bascuñán, A. 2004. The Influence of Wind on radiata Pine Tree Shape and Wood Stiffness. Thesis for the Degree of Masters of Forestry Science. University of Canterbury, New Zealand. 122 p.

Cardoso, A.; Trugilho, P.; Lima, J.; Rosado, S.; Mendes, L. 2005. Longitudinal residual strain in different spacing and ages in hybrid clone of Eucalyptus. Cerne 11: 218-224.

Carter, P.; Briggs, D.; Ross, R.; Wang, X. 2005. Acoustic testing to enhance western forest values and meet customer wood quality needs. General Technical Report Pacific Northwest Research Station; PNW-GTR-642. Productivity of Western Forests: A Forest Products Focus pp. 121-129.

Celhay, J.A.; Bonnefoy, P.; Riquelme, F. 1999. Efecto de la intensidad de poda sobre el crecimiento de E. nitens. En actas de Silvotecna XII, simposio IUFRO: Realidad y potencial de Eucalipto en Chile: cultivo silvícola y su uso industrial. Concepción, Chile. 119-138.

Chauhan, S. 2004. Selecting and/or processing wood according to its processing characteristics. Thesis Doctor of Philosophy in forestry. School of Forestry. University of Canterbury. New Zealand.

Chauhan, S.S.; Walker, J. 2006. Variations in acoustic velocity and density with age, and their interrelationships in radiata pine. Forest Ecology and Management 229: 388-394.

Cremer, K.; Borough, C.; Mckinnell, F.; Carter, P. 1982. Effects of stocking and thinning on wind damage in plantations. N.Z.J. For. Sci. 12(2): 244-268.

De Fégely, R. 2004. Sawing regrowth and plantation hardwoods with particular reference to growth stresses. Part A Literature review. Report Project no. PN02.1308. Forest and Wood Products Research Development Corporation.

Farrell, R.; Innes, T.; Nolan, G. 2008. Sorting plantation Eucalyptus nitens logs with acoustic wave velocity. FWPRDC project PN07.3018. Forest and Wood Products Australia. 27 p.

Farrell, R.; Atyeo, W.; Siemon, G.; Daian, G.; Ozarska, B. 2010. Impact of sapwood and the properties and market utilization of plantation and young hardwoods: Executive Summary and Literature Review (PART A). Project number: PNB039-0708. Forest and Wood Products Australia. 81 p.

Ferrand, J.C. 1983. Growth stresses and silviculture of eucalypts. Australian Forest Research 13: $75-81$.

Ferreira, M. 1968. Estudio da variacao da densidade basica da Madeira de Eucalyptus alba and Eucalyptus saligna. Piracicaba, Brasil. 77 p.

Grabianowski, M. 2003. Measuring acoustic properties in lumber and trees. MSc thesis, University of Canterbury, Christchurch, New Zealand. 148 p. 
Guimaraes, R.F. 1965. Observation on diameters, heights, survival and weight of the wood in Eucalyptus saligna at various initial spacings. An Bras Flor Inst Nac Pinho 17:31-45.

Higgs, M.L. 1969. Genetic and environmental factors influencing commercially important wood properties of Eucalyptus grandis. PhD Thesis, Australian National Univ. Canberra. Australia.

INFOR. Instituto Forestal. 2007. Proyecto "Desarrollo de opciones productivas de mayor valor para plantaciones de Eucalyptus nitens en la IX y X regiones: Propuesta silvícola”. [en línea]. Disponible en <http://www.infor.cl/nitens/p2antedecentes.html> [consultado Noviembre 2010].

INFOR. Instituto Forestal. 2010. Anuario Forestal 2010. Boletín Estadístico Nº 128. 134 p.

Kennedy, R.W. 1995. Coniferous wood quality in the future: concerns and strategies. Wood Sci. Technol. 29(5): 321-338.

Kenneth, R. 2001. A general theory for the origin of growth stresses in reaction wood: how trees stay upright. IAWA Journal 22 (3): 205-212.

Lasserre, J.P.; Mason, E.; Watt, M. 2005. The effects of genotype and spacing on Pinus radiata D. Don corewood stiffness in an 11-year old experiment. Forest Ecology and Management 205: 375-383.

Lasserre, J.P.; Mason, E.; Watt, M. 2008. Influence of the main and interactive effects of site, stand stocking and clone on Pinus radiata D. Don corewood modulus of elasticity. Forest Ecology and Management 255: 3455-3459.

Medhurst, J.; Beadle, C. 2000. Thinning for solid Wood products in Eucalyptus nitens plantations. In: The Future of Eucalypts for Wood Products. Eds. L Henderson, G Waugh, G Nolan and P Bennett. Proceedings of IUFRO Conference, Launceston, Australia. 343-348.

Medhurst, J.; Beadle, C.; Neilsen, W. 2001. Early-age and later-age thinning affects growth, dominance, and intraspecific competition in Eucalyptus nitens plantations. Can. J. For. Res. 31: 187-197.

Medhurst, J.; Battaglia, M.; Beadle, C. 2002. Measured and predicted changes in tree and stand water use following high-intensity thinning of an 8-year-old Eucalyptus nitens plantation. Tree Physiol. 22:775-784.

Megraw, R.A. 1986. Douglas-fir wood properties. In Oliver C, D Hanley, J Johnson eds. Douglasfir: Stand management for the future. Inst. of For. Res. Contrib. 55. College of Forest Resources, University of Washington, Seattle. p. 81-96.

Muñoz, F.; Espinosa, M.; Herrera, M.; Cancino, J. 2005. Características del crecimiento en diámetro, altura y volumen de una plantación de Eucalyptus nitens sometida a tratamientos silvícolas de poda y raleo. Bosque 26(1): 93-99.

Muñoz, F.; Neira, A.; Cancino, J. 2010. Efecto del raleo en la densidad básica de la madera de Eucalyptus nitens (Deane \& Maiden) Maiden. Interciencia 35(8): 581-585.

Mutizabal, A. 2007. Comportamiento de tensiones de crecimiento en tres procedencias de Eucalyptus nitens, Región del Bío-Bío. Memoria para optar al título de Ingeniero Forestal, Facultad de Ciencias Forestales, Universidad Austral de Chile. Valdivia. Chile. 57 p. 
Neilsen, W.A.; Pinkard, E. 1999. Developing silvicultural regimes for saw log and veneer production from temperate eucalypt plantations in Tasmania. In: Proceedings of the Conference on XII SILVOTECNA. Eucalypts in Chile, Present and Future. Concepción, Chile, p. 27.

Nolan, G.; Greaves, B.; Washusen, R.; Parsons, M.; Jennings, S. 2005. Eucalypt plantations for solid wood products in Australia - A review: If you don't prune it, we can't use it. Project no: PN04.3002. Forest \& Wood Products Research \& Development Corporation Victoria, Australia. 130 p.

Nutto, L.; Touza, M. 2004. High quality Eucalyptus saw log production. In Eucalyptus in a changing world. Proceedings of International Union of Forestry Research Organisations Conference, 1115 October. Aveiro, Portugal. pp. 658-666.

Prodan, M.; Peters, R.; Cox, F.; Real, P. 1997. Mensura Forestal. Serie Investigación y Educación en Desarrollo Sostenible. IICA- BMZ/GTZ. 561 p.

Raymond, C.; Kube, P.; Pinkard, L.; Savage, L.; Bradley, A. 2004. Evaluation of non-destructive methods of measuring growth stress in Eucalyptus globulus: Relationships between strain, wood properties and stress. Forest Ecology and Management 190: 187-200.

Roth, B.; Li, X.; Huber, D.; Peter, G. 2007. Effects of management intensity, genetics and planting density on wood stiffness in a plantation of juvenile loblolly pine in the southeastern USA. Forest Ecology and Management 246: 155-162.

Sarén, M.; Serimaa, R.; Andersson, S.; Saranpää, P.; Keckes, J.; Fratzl, P. 2004. Effect of growth rate on mean microfibril angle and cross-sectional shape of tracheids of Norway spruce. Trees 18: 354-362.

SAS INSTITUTE INC. 2000. SAS/STAT User's Guide: Version 8, vol. 1-3. SAS Institute Inc. Cary, NC. 3884 p.

Schlatter, J.; Gerding, V.; Adriazola, J. 1994. Sistema de ordenamiento de la tierra. Herramienta para la planificación forestal aplicado a las Regiones VII, VIII y IX. Facultad de Ciencias Forestales, Universidad Austral de Chile. 33 p.

Schönau, A.P. 1974. The effect of planting space and pruning on growth, yield and timber density of Eucalyptus grandis. S Afr For J. 88:16-23.

Stöckle, M. 1996. Efecto inicial de poda y raleo en el crecimiento de un rodal de Eucalyptus nitens de 6 años de edad. Tesis Ingeniero Forestal. Facultad de Ciencias Forestales, Universidad de Concepción. Concepción, Chile. 26 p.

Telewski, F.W. 1995. Wind-induced physiological and developmental responses in trees. Wind and Trees. Ed. Coutts-MP and Grace-J. Cambridge University Press. p 237-263.

Trugilho, P.; Iwakiri, S.; Rocha, M.; Matos, J.; Saldanha, L. 2004. Age and diametric class effects on longitudinal residual strain in Eucalyptus dunnii trees. Revista Arvore 28: 725-731.

Trugilho, P.; Lima, J.; De Pádua, F.; De Carvalho, L.; Andrade, C. 2006. Deformação residual longitudinal (DRL) e tangencial (DRT) em seis clones de Eucalyptus spp. Cerne 12: 279-286. 
Valdés, R. 2004. Determinación de tensiones crecimiento de Eucalyptus nitens mediante método no destructivo. Memoria para optar al título de Ingeniero en Industrias de la Madera, Universidad de Talca, Chile. 48 p.

Valencia, J. 2008. Application of non-destructive evaluation techniques to the prediction of solid-wood suitability of plantation grown Eucalyptus nitens logs. Thesis Submitted in fulfillment of the requirements for the Degree of Master of Science. University of Tasmania, Australia.

Valencia, J.; Harwood, C.; Washusen, R.; Morrow, A.; Wood, M.; Volker, P. 2011. Longitudinal growth strain as a log and wood quality predictor for plantation-grown Eucalyptus nitens sawlogs. Wood Sci Technol 45:15-34.

Waghorn, M.J.; Mason, E.; Watt, M. 2007. Influence of initial stand density and genotype on longitudinal variation in modulus of elasticity for 17-year-old Pinus radiata. For Ecol Manag 252:6772 .

Warren, E.; Smith, R.; Apiolaza, L.; Walker, J. 2009. Effect of stocking on juvenile wood stiffness for three Eucalyptus species. New Forests 37: 241-250.

Watt, M.S.; Moore, J.; Facon, J.; Downes, G.; Clinton, P.; Coker, G.; Davis, M.; Simcock, R.; Parfitt, R.; Dando, J.; Mason, E.; Bown, H. 2006. Modeling the influence of stand structural, edaphic and climatic influences on juvenile Pinus radiata dynamic modulus of elasticity. For. Ecol. Manage. 229: 136-144.

Wilson, J.S.; Oliver, C. 2000. Stability and density management in Douglas-fir plantations. Can. J. For. Res. 30: 910-920.

Yang, J.L.; Evans, R. 2003. Prediction of MOE of eucalypt wood from microfibril angle and density. Holz als Roh - und Werkstoff 61: 449-452.

Yang, J.L. 2005. The impact of log-end splits and spring on sawn recovery of 32-year-old plantation Eucalyptus globulus Labill. Holz als Roh - und Werkstoff 63: 442-448.

Yang, J.L.; Baillères, H.; Okuyama, T.; Muneri, A.; Downes, G. 2005. Measurement methods for longitudinal surface strain in trees: a review. Australian Forestry 68(1): 34-43.

Zobel, B.; Van Buijtenen, J. 1989. Wood variation. Its causes and control. Springer Series in Wood Science. Springer-Verlag. Berlin. 363 p.

Zobel, B.; Sprague, J. 1998. Juvenile Wood in Forest Trees. Ediciones Springer. Berlín, Alemania. 300 p. 